\title{
Modeling of Detection Limit for Competitive Immunoassay Using Surface Plasmon Resonance Sensor
}

\author{
Masahiro Naruse, ${ }^{*}$ Masahiro Morishita, Takeshi Onodera, ${ }^{1}$ \\ Kiyoshi Toko, ${ }^{1}$ and Yuzuru Hayashi ${ }^{2}$ \\ Ground Systems Research Center, Acquisition, Technology \& Logistics Agency, \\ 2-9-54 Fuchinobe, Chuou-ku, Sagamihara, Kanagawa 252-0206, Japan \\ ${ }^{1}$ Research and Development Center for Taste and Odor Sensing, Kyushu University, \\ W2-455, 744 Motooka, Nishi-ku, Fukuoka 819-0395, Japan \\ ${ }^{2}$ Teikyo Heisei University, Faculty of Pharmaceutical Sciences, \\ 4-21-2 Nakano, Nakano-ku, Tokyo 164-8530, Japan \\ (Received November 30, 2015; accepted February 8, 2016)
}

Keywords: surface plasmon resonance sensor, trinitrotoluene, detection limit modeling, environmental influence, improvised explosive devise

In this study, we investigated the detection limit of a competitive immunoassay using a surface plasmon resonance (SPR) sensor by both experimental and theoretical approaches. Highly sensitive explosive detection is required to prevent damage from hidden explosives such as improvised explosive devices (IED), and therefore in this study we focused on trinitrotoluene (TNT) detection using an anti-TNT antibody. As an experimental approach, competitive immunoassay experiments were conducted using a published technique for TNT detection, and the results were statistically analyzed to estimate the detection limit according to a reported method for an enzyme-linked immunosorbent assay (ELISA). In addition, a mathematical model describing measurement errors was developed and the detection limit was determined based on the correlated data between the SPR sensor response and antibody binding obtained from the experiments. Both approaches showed that the detection limit is about $10 \mathrm{ppb}(\mathrm{ng} / \mathrm{mL})$, and hence the theory is in good agreement with the experiment. Finally, using our mathematical model, we proposed a method for determining the detection limit in the presence of environmental influences.

\section{Introduction}

An immunoassay, the surface plasmon resonance (SPR) immunosensor, is known to possess high sensitivity; however, only a validation using the standard deviation (SD) has been adopted for the estimation of detection capabilities. ${ }^{(1-4)}$ The $95 \%$ confidence intervals of an estimated SD are $\sim 20 \%$ of the true value for $n=40$, but $\sim 60 \%$ for $n=6{ }^{(5)}$ and the number of repetitions required is unrealistic. Mathematical modeling of an enzyme-linked immunosorbent assay (ELISA) enabled a precise estimation of a detection limit in a previous study. ${ }^{(5)}$ In the study, the detection capability of ELISA was estimated using the relative standard deviation (RSD), as done in related methods in chromatography and spectrometry. ${ }^{(6-18)}$ The procedure was standardized by the International Organization for Standardization (ISO) and Japan Industrial Standards (JIS), ${ }^{(19,20)}$ and the detection

${ }^{\bar{*}}$ Corresponding author: e-mail: naruse.masahiro.qb@cs.alta.mod.go.jp 
limit and the quantitation limit are defined as the concentration with 30\% RSD and 10\% RSD, respectively. Thus we applied this mathematical modeling to the competitive immunoassay of TNT using an SPR immunosensor. Since the errors from various factors are independent of each other, the error parameters derived in this paper could be applicable to other detections with SPR immunosensors.

Explosive detection is important for counter-terrorism by both commercial and military technologies, since threats from improvised explosive devices (IEDs) have increased in recent years. IED is a generic term used to refer to nonindustrially fabricated explosive material that takes various forms; therefore, the detection of IEDs by their shape is difficult in terms of its reliability. For trace detection, chemical or mass characteristics of explosives can be utilized. For chemical detection, products using color reactions have been widely used in the field. ${ }^{(21-23)}$ Lately, for mass detection, ion mobility spectrometry (IMS) technologies have been put to practical use for explosive detection, and IMS products have been placed in various spots, such as airports. ${ }^{(24-27)}$ Durring the fabrication process, several nanograms or more of explosives may attach to the surface of an IED, and a sensor has to detect this minor amount of explosive in the field for successful IED screening. Color reactions are vulnerable to contamination and are less sensitive. On the other hand, IMS is sensitive and less vulnerable to contamination. Thus, sensor technology with sensitivity, specificity, and resistance to contamination is needed for IED detection, and quantitative measurement is desirable for the separation of analyte signals and environmental influences.

The purpose of this study is to propose the mathematical modeling of competitive immunoassay using SPR for TNT as a typical military explosive. SPR immunoassay for TNT has been previously reported as a highly sensitive detection method. ${ }^{(1,4)}$ At first for simplicity, the relationship between the SPR sensor response and antibody binding was examined. Then, a competitive immunoassay for TNT was carried out, and a theoretical model was constructed. An elaboration of the SPR immunoassay using our mathematical model is expected to improve counter-IED technology.

\section{Materials and Methods}

\subsection{Theory}

All symbols in the following equations are listed in Table 1.

Table 1

Each symbol in the left column indicates the corresponding description in right column.

\begin{tabular}{ll}
\hline$X$ & TNT concentration \\
$Y$ & Antibody concentration \\
$Y_{\mathrm{F}}$ & Free antibody concentration \\
$\Delta R_{\max }$ & Measurement (maximum SPR sensor response) \\
$\rho_{\mathrm{T}}$ & Total RSD of errors in measuring \\
$\rho_{\mathrm{X}}$ & RSD of input concentration of TNT \\
$\rho_{\mathrm{Y}}$ & RSD of input concentration of antibody \\
$\sigma_{\mathrm{L}}$ & SD of sensor fluctuation \\
$k_{\mathrm{a}}, k_{\mathrm{b}}, k_{\mathrm{c}}$ & Coefficient of SPR response modeling \\
$K$ & Coefficient of balanced equation \\
\hline
\end{tabular}




\subsubsection{Modeling the relationship between SPR sensor response and antibody binding}

First, we focused on the relationship between the SPR sensor response and antibody binding. Since antibody concentration $Y$ equals free antibody concentration $Y_{\mathrm{F}}$ under conditions without antigen, a basic formula for $Y_{\mathrm{F}}$ was derived. As SPR sensor responses are not proportional to the antibody concentration, Eq. (1), using a logistic function on the logarithmic $X$-axis, represents the relationship between SPR sensor response $\Delta R_{\max }$ and $Y_{\mathrm{F}}$ (see Fig. 1).

$$
\Delta R_{\max }=\frac{k_{\mathrm{c}}}{1+k_{\mathrm{a}} Y_{\mathrm{F}}^{-k}}
$$

where the coefficients $k_{\mathrm{c}}$ and $k_{\mathrm{b}}$ can be determined by the maximum SPR sensor response and the concentration corresponding to half of the maximum SPR sensor response. Then, coefficient $k_{\mathrm{a}}$ can be derived using the least-squares fitting method.

Based on a previous study of ELISA, ${ }^{(5)}$ using the error propagation rule, Eq. (2) was derived from Eq. (1), denoting the total RSD of the measurements $\rho_{\mathrm{T}}$ (see Appendix A).

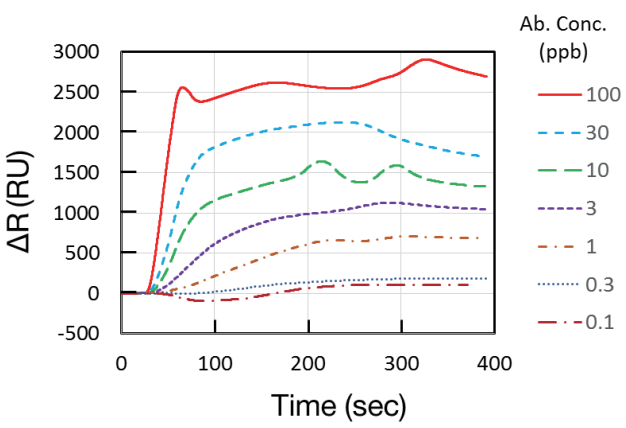

(a)

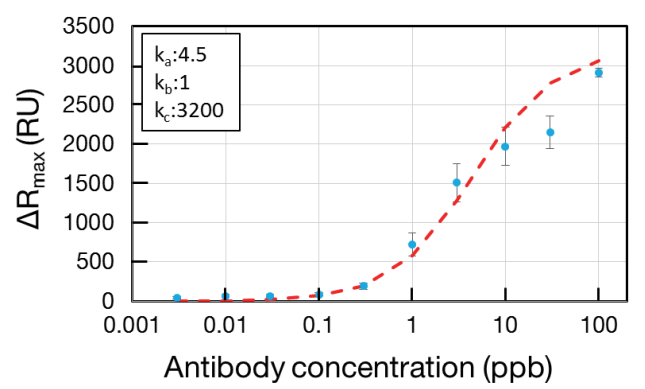

(c)

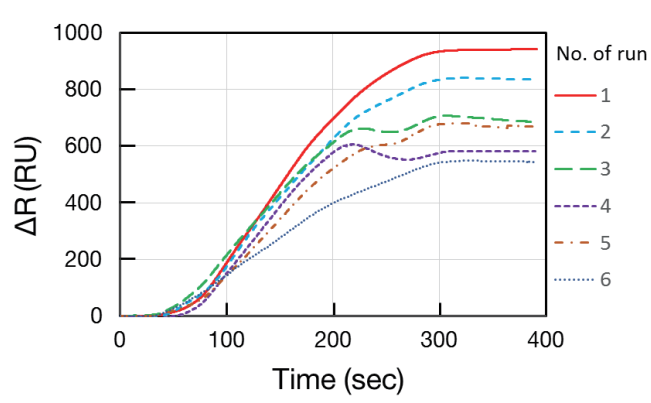

(b)

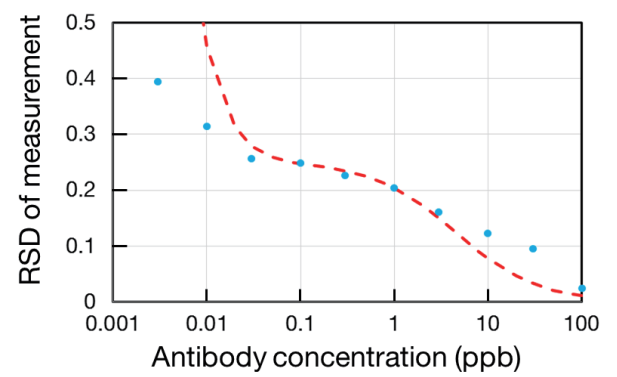

(d)

Fig. 1. (Color online) Relationship between the SPR sensor response and the antibody concentration. (a) Representative SPR sensor responses to each antibody concentration. (b) Comparison of SPR sensor responses of six repeated experiments for $1 \mathrm{ppb}$ antibody concentration. (c) Correlation between measurements and antibody concentrations. Data points and error bars, averaged measurements, and SDs $(n=6)$; broken line, the theoretical curve [Eq. (1)]. (d) Correlation between RSDs of measurements and antibody concentrations. Data points, the experimental RSD of measurements; broken line, the theoretical RSD of measurements [Eq. (2)]. 


$$
\rho_{\mathrm{T}}^{2}=\frac{k_{\mathrm{a}}^{2} k_{\mathrm{b}}^{2}}{\left(1+k_{\mathrm{a}} Y_{\mathrm{F}}^{-k_{\mathrm{b}}}\right)^{2}} Y_{\mathrm{F}}^{-2 k_{\mathrm{b}}} \rho_{\mathrm{Y}}^{2}+\frac{\left(1+k_{\mathrm{a}} Y_{\mathrm{F}}^{-k_{\mathrm{b}}}\right)^{2}}{k_{\mathrm{c}}^{2}} \sigma_{\mathrm{L}}^{2}
$$

Equation (2) includes major error factors, namely the pipetting and injecting of the viscous antibody solution and internal fluctuations of the SPR sensor; $\rho_{\mathrm{Y}}$ denotes the RSD of the concentration of the antibody solution, and $\sigma_{\mathrm{L}}$ denotes the background $\mathrm{SD}$ of the SPR sensor response.

\subsubsection{Modeling the competitive immunoassay}

In competitive immunoassay, determining the antigen (TNT) concentration $X$ is the purpose of the measurement. The value of $X$ influences the ratio of the concentrations of free antibody to whole antibody, and Eq. (3) is the balanced equation of binding describing the relationship between $X$, $Y$, and $Y_{\mathrm{F}}$ (see Appendix B).

$$
Y_{\mathrm{F}}=\frac{K Y}{X+K}
$$

Although the antibody possesses two binding sites, the model is simplified by considering it to be monovalent. Free antibody concentration $Y_{\mathrm{F}}$ can be calculated from TNT concentration $X$, antibody concentration $Y$, and the coefficient $K$. Substituting Eq. (3) into $Y_{\mathrm{F}}$ in Eq. (1), we obtain a model for competitive immunoassay as Eq. (4), and the coefficient $K$ is given by the least-squares fitting method using experimental data [see Fig. 2(c)].

$$
\Delta R_{\max }=\frac{k_{\mathrm{c}}}{1+k_{\mathrm{a}}\left(\frac{K Y}{X+K}\right)^{-k_{\mathrm{b}}}}
$$

Derivation of a theoretical detection limit was based on the previous study and the international standard of ISO. ${ }^{(5,19)}$ For the derivation, the RSD of the estimated concentration of TNT is needed. Equation (5), derived from Eq. (4) in a similar manner to Eq. (2) (see Appendix C), indicates the total RSD of the measurements, considering not only the RSD of the injected volume of antibody $\left(\rho_{\mathrm{Y}}\right)$, but also of TNT as the analyte $\left(\rho_{\mathrm{X}}\right)$.

$$
\rho_{\mathrm{T}}^{2}=\frac{k_{\mathrm{a}}^{2} k_{\mathrm{b}}^{2}\left(\frac{K Y}{X+K}\right)^{-2 k_{\mathrm{b}}}}{\left(1+k_{\mathrm{a}}\left(\frac{K Y}{X+K}\right)^{-k_{\mathrm{b}}}\right)^{2}} \rho_{\mathrm{Y}}^{2}+\frac{k_{\mathrm{a}}^{2} k_{\mathrm{b}}^{2} X^{2}\left(\frac{K Y}{X+K}\right)^{-2 k_{\mathrm{b}}}}{(X+K)^{2}\left(1+k_{\mathrm{a}}\left(\frac{K Y}{X+K}\right)^{-k_{\mathrm{b}}}\right)^{2}} \rho_{\mathrm{X}}^{2}+\frac{\left(1+k_{\mathrm{a}}\left(\frac{K Y}{X+K}\right)^{-k_{\mathrm{b}}}\right)^{2}}{k_{\mathrm{c}}^{2}} \sigma_{\mathrm{L}}^{2}
$$

As in the previous study, ${ }^{(5)}$ the RSD of measurements can be transformed to the RSD of the estimated concentrations by dividing by the slope of the calibration curve. The detection limit was given as a concentration with $30 \%$ RSD of the concentration estimates in both experimental and theoretical approaches. 


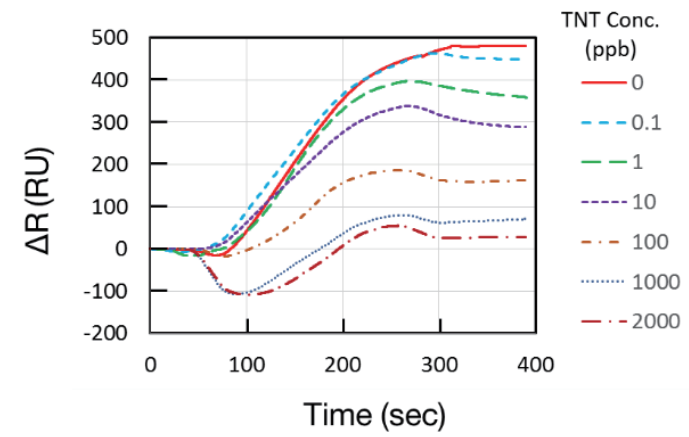

(a)

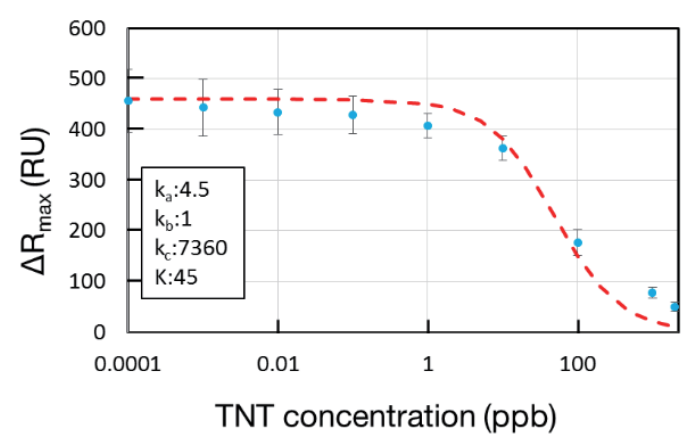

(c)

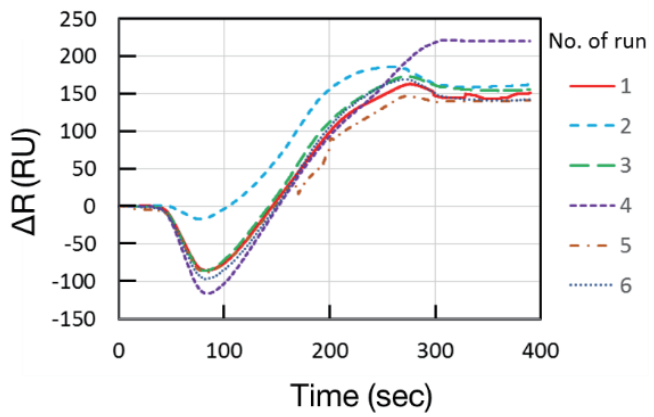

(b)

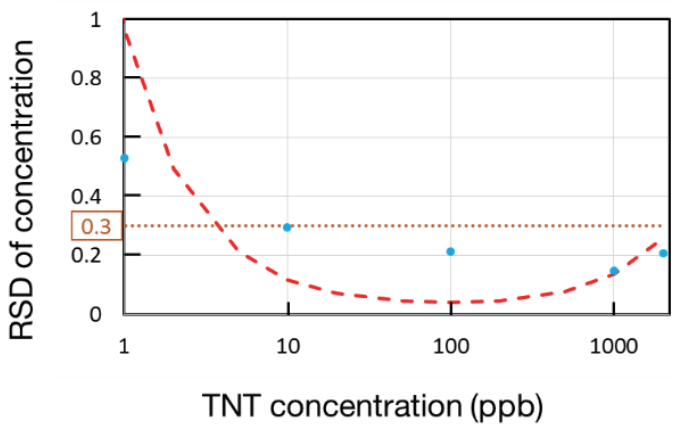

(d)

Fig. 2. (Color online) Results of competitive immunoassay for TNT. (a) Representative SPR sensor responses to each TNT concentration. (b) Comparison of SPR sensor responses of six repeated experiments for $100 \mathrm{ppb}$ TNT concentration. (c) Correlation between measurements and TNT concentration in the competitive immunoassay. Data points and error bars, averaged measurements, and SDs $(n=6)$; broken line, the theoretical curve calculated from Eq. (4). (d) Correlation between RSDs of the estimated concentrations and TNT concentrations. Data points, the experimental RSDs of the estimated concentration; line, the theoretical RSD of the estimated concentration calculated from Eqs. (4) and (5).

\subsection{Experimental procedure}

\subsubsection{SPR measurements}

The target analyte was TNT (Chugoku Kayaku Co., Ltd.). For the SPR measurements, RANA (Kyushu Keisokki Co., Ltd.) and RN-cp-TNT (Kyushu Keisokki Co., Ltd.) were used as the SPR immunosensor and its sensor chip, respectively; dinitrophenyl (DNP)-glycine was immobilized on the surface of RN-cp-TNT by the same procedure as "EG 17 (8:2) chip" in the previous study.(1) The antibody solution, Qatb-TNT01 (Kyushu Keisokki Co., Ltd.), contained the identical anti-TNT antibody as used in the previous study. ${ }^{(28)}$

SPR measurements using an indirect competitive method were performed as previously described.(1,4) Experiments for finding the correlation between the SPR sensor responses and antibody concentrations were carried out six times for each antibody concentration in a range from $3 \mathrm{ppt}$ to $100 \mathrm{ppb}(\mathrm{ng} / \mathrm{mL})$. Experiments for competitive immunoassay were implemented six times for each TNT concentration in a range from $0.1 \mathrm{ppb}$ to $2 \mathrm{ppm}$, with the antibody concentration fixed at $0.3 \mathrm{ppb}$. 


\subsubsection{Data analysis}

The RSD of measurements was obtained simply by dividing the SD of the measurements by the mean of measurements for each condition. The derivation of the detection limit was based on the previous study and the international standard of ISO. ${ }^{(5,19)}$ At first all measurements were transformed to estimated TNT concentrations using the calibration curve described by Eq. (4); then the RSD of the estimated concentration was obtained by dividing the SD of the estimated concentration by the mean of the estimated concentration for each condition.

\section{Results and Discussion}

\subsection{Relationship between the SPR sensor response and antibody binding}

Figure 1(a) shows typical examples of SPR sensor responses at each antibody concentration, and Fig. 1(b) shows six runs at $1 \mathrm{ppb}$ antibody concentration. Responses increased with increasing antibody concentration, and the time course was slightly varied for each run. Plots with error bars in Fig. 1(c) denote the relationship between the SPR sensor response and the antibody concentration. In the case of the ELISA measurement, the measured fluorescent intensities were proportional to the antibody concentration, whereas in contrast, SPR sensor responses were not proportional to the TNT concentration. Thus a logistic function described by Eq. (1) was used for our modeling [broken line in Fig. 1(c)].

To validate the modeling, RSDs of measurements calculated from the model [broken line in Fig. 1(d)] were compared with those from the experiment [plots in Fig. 1(d)]. In the model calculation, the SD of errors arising from the instrument, $\sigma_{\mathrm{F}}=0.000271$ in Eq. (2), was given as the SD of measurements in the experiment injecting only buffer instead of the antibody solution, and the RSD in the preparation of the antibody solution, $\rho_{\mathrm{Y}}=0.25$ in Eq. (2), was determined by considering errors associated with the injection error and adsorption to the connection tube of the SPR sensor leading from the fabricant. As shown in Fig. 1(d), the model-based calculation and experimental results exhibited similar patterns in response to changes in the antibody concentration, although there are some differences at higher concentrations because of the misfit of modeling as shown in Fig. 1(a). Therefore, these results suggest that our model simulates the relationship between SPR sensor response and antibody binding with accuracy, although the values of $k_{\mathrm{c}}$ have to be calibrated according to the response of each sensor chip.

\subsection{Evaluation of detection limit in competitive immunoassay}

Figure 2(a) shows typical examples of SPR sensor responses at TNT concentrations ranging from $3 \mathrm{ppt}$ to $100 \mathrm{ppb}$ in the competitive immunoassay experiment, and Fig. 2(b) shows six runs at $100 \mathrm{ppb}$ TNT concentration. Responses decreased with increasing TNT concentration, and the time course was slightly varied for each run. Data points and error bars in Fig. 2(c) denote the results of competitive immunoassay for $0.3 \mathrm{ppb}$ antibody concentration; SPR sensor responses decrease significantly from 1 to $1000 \mathrm{ppb}$ of TNT. Using Eq. (4), SPR sensor response in the competitive immunoassay was calculated [broken line in Fig. 2(c)]. Comparison of experimental and theoretical results indicated that the experimental result can be interpreted by our model. 
The RSD of the estimated TNT concentration was calculated for the estimation of the detection limit $\left(\rho_{\mathrm{X}}=0.25\right.$; same as $\left.\rho_{\mathrm{Y}}\right)$; data points in Fig. 2(d) are from experimental data, and the broken line is calculated from the theoretical model. Although there are some differences in RSD for 10 to $100 \mathrm{ppb}$ concentration, in totality, the theoretical line almost traces the plots. The dotted line in Fig. 2(d) indicates RSD $=0.3$, as a detection limit threshold, indicating that the experimental detection limit is about $10 \mathrm{ppb}$, and the theoretical detection limit is about $5 \mathrm{ppb}$. Therefore, our mathematical model could predict the detection limit of the TNT detection using competitive immunoassay.

Using the mathematical model, an optimized antibody concentration can be predicted, which corresponds to a minimum detection limit. Figure 3(a) shows the correlation between the theoretical detection limit and the antibody concentration, and the minimum detection limit is 3.2 $\mathrm{ppb}$ for $5 \mathrm{ppb}$ antibody concentration. Excess antibody concentration is supposed to inhibit the competition at lower concentrations of TNT; on the other hand, a shortage of antibody seems to reduce the signal-to-noise $(\mathrm{S} / \mathrm{N})$ ratio. In Fig. 3(b), a competitive immunoassay under optimized conditions, namely for $5 \mathrm{ppb}$ antibody, resulted in a detection limit of about $3 \mathrm{ppb}$, similar to the theoretical calculation.

The proposed mathematical model seems to be valid for general immunoassays using other antibody-antigen interactions by fitting four coefficients, $k_{\mathrm{a}}, k_{\mathrm{b}}, k_{\mathrm{c}}$, and $K$. In addition to TNT, immunoassays for other explosives, cyclotrimethylen-trinitramine (RDX) and triacetone triperoxide (TATP), have been reported. ${ }^{(29-32)}$ Elaboration of the detection using our theoretical model is expected to improve these assays.

\subsection{Detection limit in the presence of environmental influences}

In the presence of environmental influences, for example suspended TNT in the atmosphere in conflict areas, the effects should be accounted for in our inspection of IED candidates. In such cases, environmental TNT must be measured as a background sample. We assume two collection methods are used for the inspection; one is a swabbing method, and the other is a suction method.

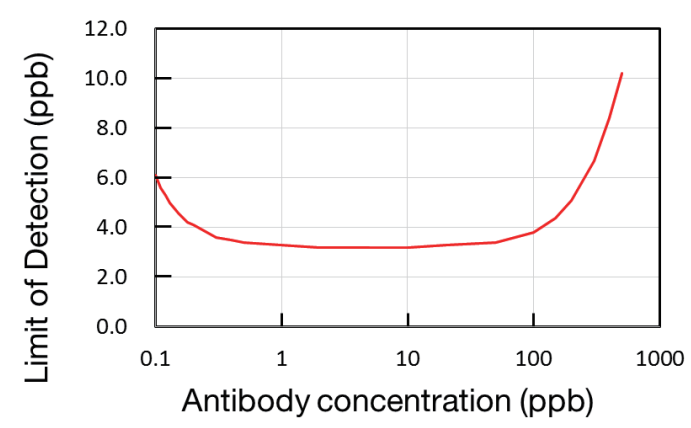

(a)

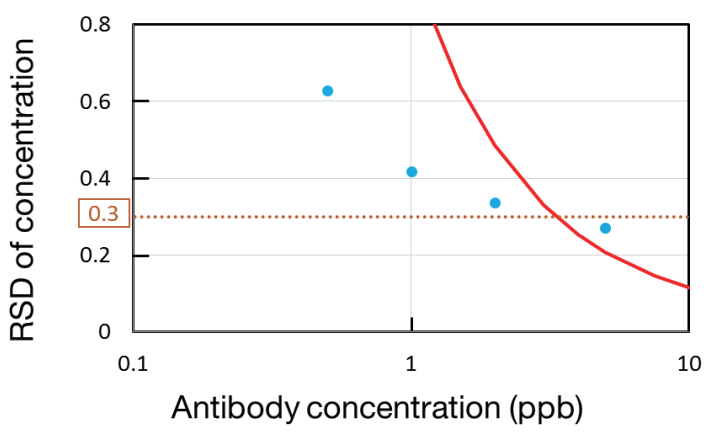

(b)

Fig. 3. (Color online) Results of optimization for antibody concentrations. (a) Correlation between the theoretical detection limit and the antibody concentration. (b) Correlation between the RSD of the estimated concentration and TNT concentration. Data points, the experimental RSD of the estimated concentration; line, the theoretical RSD of the estimated concentration calculated from Eqs. (4) and (5). 
Background samples could be obtained from swabbing a neighboring rock's surface, or suction of the atmosphere. Here, $\Delta R_{\text {env }}$ is defined as the measured value of a blank sample. Using Eq. (4), both the estimated concentration $X_{\text {env }}$ and the RSD of the estimated concentration $\rho_{\text {env }}$ can be calculated from $\Delta R_{\text {env. }}$ Equation (6), derived from Eq. (5), represents the RSD of the measurements associated with the environmental influence as the last term.

$$
\rho_{\mathrm{T}}^{2}=\frac{k_{\mathrm{a}}^{2} k_{\mathrm{b}}^{2}\left(\frac{K Y}{X+K}\right)^{-2 k_{\mathrm{b}}}}{\left(1+k_{\mathrm{a}}\left(\frac{K Y}{X+K}\right)^{-k_{\mathrm{b}}}\right)^{2}}\left(\rho_{\mathrm{Y}}^{2}+\frac{X^{2} \rho_{\mathrm{X}}^{2}}{(X+K)^{2}}\right)+\frac{\left(1+k_{\mathrm{a}}\left(\frac{K Y}{X+K}\right)^{-k_{\mathrm{b}}}\right)^{2}}{k_{\mathrm{c}}^{2}} \sigma_{\mathrm{F}}^{2}+\rho_{\mathrm{env}}^{2}
$$

Using $\rho_{\text {env }}, X=X_{\mathrm{c}}$ can be calculated with $\rho_{\mathrm{T}}=0.3$ in Eq. (6), and then $X_{\mathrm{DL}}$ satisfies Eq. (7) and is a detection limit. Therefore, $\Delta R_{\mathrm{T}}$ calculated back from $X_{\mathrm{c}}$ using Eq. (4) should be a threshold for the detection.

$$
X_{\mathrm{DL}}=X_{\mathrm{c}}-X_{\mathrm{env}}
$$

\section{Conclusions}

In this study, a model describing SPR responses and detection limits was proposed, which enables the prediction of detection limit for more reliable detection; it can be applied to other SPR immunoassay analyses. We proposed a method for determining the detection limit in the presence of environmental influences.

\section{Acknowledgements}

We acknowledge Kyushu Keisokki Co., Ltd., for their assistance in adjusting the SPR immunosensor.

\section{References}

1 Y. Mizuta, T. Onodera, P. Singh, K. Matsumoto, N. Miura, and K. Toko: Biosens. Bioelectron. 24 (2008) 191.

2 P. Singh, T. Onodera, Y. Mizuta, K. Matsumoto, K. Miura, and K. Toko: Sens. Actuators, B 137 (2009) 403.

3 T. Onodera, Y. Mizuta, K. Horikawa, P. Singh, K. Matsumoto, N. Miura, and K. Toko: Sens. Mater. 23 (2011) 39.

4 T. Onodera and K. Toko: Sensors 14 (2014) 16586.

5 Y. Hayashi, R. Shibata, T. Maitani, K. Imai, W. Nishimura, K. Ito, and M. Maeda: Anal. Chem. 76 (2004) 1295.

6 C. T. J. Alkemade, W. Snelleman, G. D. Boutilier, B. D. Pollard, J. D. Winefordner, T. L. Chester, and N. Omenetto: Spectrochim. Acta 33B (1978) 383.

7 G. D. Boutilier, B. D. Pollard, J. D. Winefordner, T. L. Chester, and N. Omenetto: Spectrochim. Acta 33B (1978) 401.

8 P. W. J. M. Boumans: Spectrochim. Acta 46B (1991) 917.

9 H. C. Smit and H. L. Walg: Chromatographia 9 (1976) 483.

10 T. J. Bahowick and R. E. Synovec: Anal. Chem. 67 (1995) 631.

11 Y. Hayashi and R. Matsuda: Anal. Chem. 66 (1994) 2874.

12 R. Matsuda, Y. Hayashi, S. Sasaki, K. Saito, K. Iwaki, H. Harakawa, M. Satoh, Y. Ishizuki, and T. Kato: Anal. Chem. 70 (1998) 319. 
E. D. Prudnikov: Fresenius J. Anal. Chem. 337 (1990) 412.

P. W. J. M. Boumans: Anal. Chem. 66 (1994) 459.

15 E. Grushka and I. Zamir: Chem. Anal. 34 (1989) 529.

16 N. W. Bower and J. D. Ingle Jr.: Anal. Chem. 48 (1976) 686.

17 N. W. Bower and J. D. Ingle Jr.: Anal. Chem. 49 (1977) 574.

18 J. D. Ingle Jr. and S. R. Crouch: Spectrochemical Analysis (Prentice-Hall, New Jersey, 1988).

19 International Standard 2008, ISO 11843-5.

20 Japanese Industrial Standard 2011, JIS Z 8462-5.

21 J. Nakamura, T. Ohta, A. Takada, and Y. Nakayama: Explosive Tracing Handbook (Maruzen Co., Ltd., Tokyo, 2010) p. 25 (in Japanese).

22 T. K. Kim, J. H. Lee, D. Moon, and H. R. Moon: Inorg. Chem. 52 (2013) 589.

23 N. Idros, M. Y. Ho, M. Pivnenko, M. M. Qasim, H. Xu, Z. Gu, and D. Chu: Sensors 15 (2015) 12891.

24 T. Maggie and H. H. Herbert Jr.: Anal. Chem. 76 (2004) 2741.

25 L. Peng, L. Hua, W. Wang, Q. Zhou, and H. Li: Scientific Reports 4 (2014) 6631.

26 D. Guo, Y. Wang, L. Li, X. Wang, and J. Luo: J. Mass Spectrom. 50 (2015) 198.

27 Q. Zhou, L. Peng, D. Jiang, X. Wang, H. Wang, and H. Li: Scientific Reports 5 (2015) 10659.

28 S. Nakamura, T. Onodera, K. Okuno, K. Toko, K. Matsumoto, and M. Iwakura: Proceedings of the 28th Sensor Symposium on Sensors, Micromachines and Applied Systems (2011) 293 (in Japanese).

29 M. A. Walter, D. Pfeifer, W. Kraus, F. Emmerling, R. J. Schneider, U. Panne, and M. G. Weller: Langmuir 26 (2010) 15418.

30 M. A. Walter, U. Panne, and M. G. Weller: Biosensors 1 (2011) 93.

31 T. Tanaka, R. Yatabe, K. Nagatomo, T. Onodera, K. Matsumoto, and K. Toko: IEEE Sensors J. 13 (2013) 4452.

32 T. Matsumoto, H. Yamaguchi, K. Kamijo, M. Akiyoshi, T. Matsunaga, and A. Harada: Bull. Chem. Soc. Jpn. 86 (2013) 198.

\section{Appendix}

\section{A. Derivation of Eq. (2)}

The total differential of the measurement, $d \Delta R_{\max }$, of Eq. (1) is

$$
d \Delta R_{\max }=\frac{\partial \Delta R_{\max }}{\partial Y_{\mathrm{F}}} d Y_{\mathrm{F}}=\frac{k_{\mathrm{a}} k_{\mathrm{b}} k_{\mathrm{c}} Y_{\mathrm{F}}^{-k_{\mathrm{b}}-1}}{\left(1+k_{\mathrm{a}} Y_{\mathrm{F}}^{-k_{\mathrm{b}}}\right)^{2}} d Y_{\mathrm{F}}=\frac{k_{\mathrm{a}} k_{\mathrm{b}} k_{\mathrm{c}} Y_{\mathrm{F}}^{-k_{\mathrm{b}}}}{\left(1+k_{\mathrm{a}} Y_{\mathrm{F}}^{-k_{\mathrm{b}}}\right)^{2}}\left(\frac{d Y_{\mathrm{F}}}{Y_{\mathrm{F}}}\right),
$$

and the variance of measurements is given as

$$
\left(d \Delta R_{\max }\right)^{2}=\frac{k_{\mathrm{a}}^{2} k_{\mathrm{b}}^{2} k_{\mathrm{c}}^{2} Y_{\mathrm{F}}^{-2 k_{\mathrm{b}}}}{\left(1+k_{\mathrm{a}} Y_{\mathrm{F}}^{-k_{\mathrm{b}}}\right)^{4}}\left(\frac{d Y_{\mathrm{F}}}{Y_{\mathrm{F}}}\right)^{2} .
$$

Dividing Eq. (A2) by the square of $\Delta R_{\max }$ [Eq. (1)], we obtain

$$
\left(\frac{d \Delta R_{\max }}{\Delta R_{\max }}\right)^{2}=\frac{k_{\mathrm{a}}^{2} k_{\mathrm{b}}^{2} Y_{\mathrm{F}}^{-2 k_{\mathrm{b}}}}{\left(1+k_{\mathrm{a}} Y_{\mathrm{F}}^{-k_{\mathrm{b}}}\right)^{2}}\left(\frac{d Y_{\mathrm{F}}}{Y_{\mathrm{F}}}\right)^{2}
$$

where $d \Delta R_{\max } / \Delta R_{\max }=\rho_{\mathrm{p}}$ is the RSD of the measurement during the preparation process, and $d Y_{\mathrm{F}} / Y_{\mathrm{F}}$ $=\rho_{\mathrm{Y}}$ denotes the RSD of random variables from handling viscous antibody solutions. 
On the other hand, the RSD of the measurement, $\rho_{\mathrm{M}}$, the measurement error arising from instruments, is given as

$$
\rho_{\mathrm{M}}^{2}=\left(\frac{\sigma_{\mathrm{F}}}{\Delta R_{\max }}\right)^{2}=\frac{\left(1+k_{\mathrm{a}} Y_{\mathrm{F}}^{-k_{\mathrm{b}}}\right)^{2}}{k_{\mathrm{c}}^{2}} \sigma_{\mathrm{F}}^{2},
$$

where $\sigma_{\mathrm{F}}$ is the noise $\mathrm{SD}$, denoting the fluctuation of the $\mathrm{SPR}$ sensor response.

Finally, total RSD of the analysis, $\rho_{\mathrm{T}}$, is given as the sum of the RSD of the preparation, $\rho_{\mathrm{p}}$, and the RSD of the measurement, $\rho_{\mathrm{M}}$.

$$
\rho_{\mathrm{T}}^{2}=\rho_{\mathrm{p}}^{2}+\rho_{\mathrm{M}}^{2}
$$

Thus, Eq. (2) can be derived from Eq. (A5).

\section{B. Derivation of Eq. (3)}

The balanced equation is represented as

$$
K=\frac{[X][Y]}{[X Y]},
$$

where $[X]$ and $[Y]$ indicate the concentrations of free antigen and antibody, respectively. The term $[X Y]$ is the concentration of antigen-antibody complex, and $K$ is the dissociation constant. The term $[Y]$ is equal to $Y_{\mathrm{F}}$, and $[X Y]$ can be described as $Y-Y_{\mathrm{F}}$. On the other hand, $[X]$ can be substituted simply by $X$, since antigen is in excess compared with antibody. Thus, Eq. (3) can be derived from Eq. (A7).

$$
K=\frac{X Y_{\mathrm{F}}}{Y-Y_{\mathrm{F}}}
$$

\section{Derivation of Eq. (5)}

The total differential of the measurement, $d \Delta R_{\max }$, of Eq. (4) is

$$
\begin{aligned}
d \Delta R_{\max } & =\frac{\partial \Delta R_{\max }}{\partial Y} d Y+\frac{\partial \Delta R_{\max }}{\partial X} d X \\
& =\frac{k_{\mathrm{a}} k_{\mathrm{b}} k_{\mathrm{c}}\left(\frac{K Y}{X+K}\right)^{-k_{b}-1}}{\left(1+k_{\mathrm{a}}\left(\frac{K Y}{X+K}\right)^{-k_{\mathrm{b}}}\right)^{2}} \frac{K}{X+K} d Y-\frac{k_{\mathrm{a}} k_{\mathrm{b}} k_{\mathrm{c}}\left(\frac{K Y}{X+K}\right)^{-k_{b}-1}}{\left(1+k_{\mathrm{a}}\left(\frac{K Y}{X+K}\right)^{-k_{\mathrm{b}}}\right)^{2}} \frac{K Y}{(X+K)^{2}} d X \\
& =\frac{k_{\mathrm{a}} k_{\mathrm{b}} k_{\mathrm{c}}\left(\frac{K Y}{X+K}\right)^{-k_{\mathrm{b}}}}{\left(1+k_{\mathrm{a}}\left(\frac{K Y}{X+K}\right)^{-k_{\mathrm{b}}}\right)^{2}} \frac{d Y}{Y}-\frac{k_{\mathrm{a}} k_{\mathrm{b}} k_{\mathrm{c}}\left(\frac{K Y}{X+K}\right)^{-k_{\mathrm{b}}}}{\left(1+k_{\mathrm{a}}\left(\frac{K Y}{X+K}\right)^{-k_{\mathrm{b}}}\right)^{2}} \frac{X}{X+K} \frac{d X}{X} .
\end{aligned}
$$


Considering the probabilistic independency of $d X$ and $d Y$, we can obtain the variance of measurements.

$$
\left(d \Delta R_{\max }\right)^{2}=\frac{k_{\mathrm{a}}^{2} k_{\mathrm{b}}^{2} k_{\mathrm{c}}^{2}\left(\frac{K Y}{X+K}\right)^{-2 k_{\mathrm{b}}}}{\left(1+k_{\mathrm{a}}\left(\frac{K Y}{X+K}\right)^{-k_{\mathrm{b}}}\right)^{4}}\left(\frac{d Y}{Y}\right)^{2}+\frac{k_{\mathrm{a}}^{2} k_{\mathrm{b}}^{2} k_{\mathrm{c}}^{2}\left(\frac{K Y}{X+K}\right)^{-2 k_{\mathrm{b}}}}{\left(1+k_{\mathrm{a}}\left(\frac{K Y}{X+K}\right)^{-k_{\mathrm{b}}}\right)^{4}} \frac{X^{2}}{(X+K)^{2}}\left(\frac{d X}{X}\right)^{2}
$$

Dividing the above equation by the square of $\Delta R_{\max }$ [Eq. (4)], we obtain

$$
\left(\frac{d \Delta R_{\max }}{\Delta R_{\max }}\right)^{2}=\frac{k_{\mathrm{a}}^{2} k_{\mathrm{b}}^{2}\left(\frac{K Y}{X+K}\right)^{-2 k_{\mathrm{b}}}}{\left(1+k_{\mathrm{a}}\left(\frac{K Y}{X+K}\right)^{-k_{\mathrm{b}}}\right)^{2}}\left(\frac{d Y}{Y}\right)^{2}+\frac{k_{\mathrm{a}}^{2} k_{\mathrm{b}}^{2}\left(\frac{K Y}{X+K}\right)^{-2 k_{\mathrm{b}}}}{\left(1+k_{\mathrm{a}}\left(\frac{K Y}{X+K}\right)^{-k_{\mathrm{b}}}\right)^{2}} \frac{X^{2}}{(X+K)^{2}}\left(\frac{d X}{X}\right)^{2},
$$

where $d \Delta R_{\max } / \Delta R_{\max }=\rho_{\mathrm{p}}$ is the RSD of the measurement during the preparation process. The terms $d Y / Y=\rho_{\mathrm{Y}}$ and $d X / X=\rho_{\mathrm{X}}$ denote the RSD of random variables from handling viscous solutions.

On the other hand, the RSD of the measurement, $\rho_{\mathrm{M}}$, the measurement error arising from instruments, is given as

$$
\rho_{\mathrm{M}}^{2}=\left(\frac{\sigma_{\mathrm{F}}}{\Delta R_{\max }}\right)^{2}=\frac{\left(1+k_{\mathrm{a}}\left(\frac{K Y}{X+K}\right)^{-k_{\mathrm{b}}}\right)^{2}}{k_{\mathrm{c}}^{2}} \sigma_{\mathrm{F}}^{2},
$$

where $\sigma_{\mathrm{F}}$ is the noise $\mathrm{SD}$, denoting the fluctuation of the SPR sensor response.

Finally, total RSD of the analysis, $\rho_{\mathrm{T}}$, is given as the sum of the RSD of the preparation, $\rho_{\mathrm{p}}$, and the RSD of the measurement, $\rho_{\mathrm{M}}$.

$$
\rho_{\mathrm{T}}^{2}=\rho_{\mathrm{p}}^{2}+\rho_{\mathrm{M}}^{2}
$$

Thus, Eq. (5) can be derived from Eq. (A12). 